\title{
Integrated modeling activities for the James Webb Space Telescope: Structural-Thermal-Optical Analysis
}

\author{
John D. Johnston ${ }^{\text {a }}$, Joseph M. Howard ${ }^{\text {a }}$ Gary E. Mosier, ${ }^{\text {}}$ Keith A. Parrish ${ }^{\text {a }}$ \\ Mark A. McGinnis, ${ }^{b}$ Marcel Bluth, ${ }^{b}$ Kevin Kim, and Kong Q. $\mathrm{Ha}^{\mathrm{c}}$ \\ ${ }^{a}$ NASA Goddard Space Flight Center, Greenbelt, MD 20771 \\ ${ }^{\mathrm{b}}$ Swales Aerospace, Beltsville, MD XXXX \\ ${ }^{c} J a c k s o n$ and Tull, Chartered Engineering, 7375 Executive Place, Seabrook MD 20706
}

\begin{abstract}
The James Web Space Telescope (JWST) is a large, infrared-optimized space telescope scheduled for launch in 2011. This is a continuation of a series of papers on modeling activities for JWST. The structural-thermal-optical, often referred to as "STOP", analysis process is used to predict the effect of thermal distortion on optical performance. The benchmark STOP analysis for JWST assesses the effect of an observatory slew on wavefront error. Temperatures predicted using geometric and thermal math models are mapped to a structural finite element model in order to predict thermally induced deformations. Motions and deformations at optical surfaces are then input to optical models, and optical performance is predicted using either an optical ray trace or a linear optical analysis tool. In addition to baseline performance predictions, a process for performing sensitivity studies to assess modeling uncertainties is described.
\end{abstract}

Keywords: JWST, thermal distortion, optics, integrated modeling, image quality, telescope

\section{INTRODUCTION}

The James Webb Space Telescope is a large, near- and mid-infrared optimized space telescope under development by a team consisting of NASA, a prime contractor team led by Northrop Grumman Space Technology (NGST), the European Space Agency (ESA), and the Canadian Space Agency (CSA). Development of the JWST is led by the JWST project at NASA Goddard Space Flight Center (GSFC). JWST will have an 18-segment, 6.5-meter primary mirror and will reside in an L2 Lissajous orbit. The observatory, Fig. 1, is composed of three main elements: an Optical Telescope Element (OTE), an Integrated Science Instrument Module (ISIM), and a Spacecraft consisting of the spacecraft bus and sunshield. The OTE is a deployable cryogenic optical system with diffraction limited performance at $2 \mu \mathrm{m}$. The OTE consists of the primary mirror segment assemblies (PMSAs), a secondary mirror support structure (SMSS), an aft optics assembly (AOS), and a backplane structure that supports the preceeding subsystems. The ISIM consists of three science instruments (Near-infrared camera, Near-infrared multi-object spectrograph, Mid-infrared camera) and a fine guidance sensor all of which are mounted to a common ISIM bench structure that is kinematically mounted to the OTE backplane. The spacecraft provides pointing and housekeeping functions for the observatory, while the deployable sunshield provides passive radiative cooling and stray light control for the telescope and science instruments.

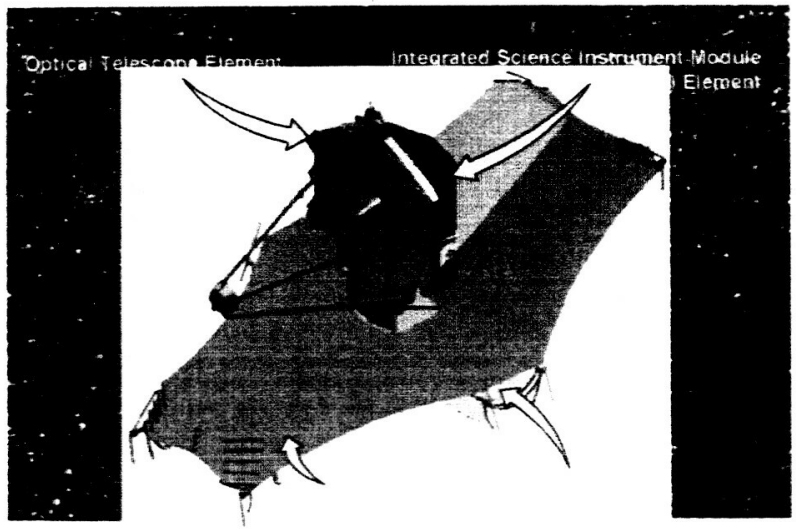

Figure 1: JWST Observatory Architecture 


\section{INTEGRATED MODELING}

Multi-disciplinary engineering analysis, or integrated modeling, is a critical element of the JWST mission. Integrated modeling primarily supports engineering design and verification of high level optical requirements for image quality and sensitivity. The image quality requirements are specified by Strehl ratio and encircled energy. Current plans for end-to-end optical performance verification rely on modeling to a degree surpassing previous programs. The final verification process requires modeling and analysis to correct for the effects of the optical test procedure, metrology, gravity, and thermal/seismic effects on opto-mechanical stability before extrapolation to behavior in the on-orbit environment.

Performance (error) budgets are maintained to control allocations to the various subsystems that comprise the JWST observatory. These budgets are traceable to the high level optical requirements. The image quality requirements are recast as requirements on wavefront error. These allocations may be roughly classified as belonging either to calibration (alignment and figuring) of the optics or to opto-mechanical stability between periodic recalibrations. The sensitivity requirement is the basis for allocations to radiometric performance, stray light suppression, and detector performance. One could view the primary integrated modeling activities as consisting of four distinct multi-disciplinary analysis efforts, three supporting verification of image quality requirements, and the fourth supporting verification of the sensitivity requirement. The analyses supporting verification of the image quality requirements are (1) thermal distortion, or STOP (Structural-Thermal-Optical), to estimate alignment figure drift due to observatory re-pointing and other transient factors, (2) jitter, to estimate the blurring and distortion due to uncompensated pointing and vibration, and (3) wavefront sensing and control, to estimate the post-calibration alignment and figure errors.

The applications of integrated modeling will change over the program life cycle. In the formulation and requirements definition phase, a strawman design was developed to address the high-level mission requirements, goals, and constraints. The role of integrated modeling was to validate this design concept by showing that the conceptual design met the requirements with margin, subject to reasonable assumptions, and that initial sub-allocations to observatory elements and sub-systems were also reasonable. Following a series of requirements reviews at the various program levels (mission, observatory, telescope, instruments), the modeling activity has aligned with the architecture/design activity in a series of cycles, of 6-9 month duration. There will be multiple design cycles between major program review milestones. At the beginning of each cycle, a baseline design (or several as long as significant design trades are active) will be "frozen", and the set of multi-disciplinary analyses will be executed to verify that the baseline design(s) for that cycle meet the optical system requirements, with margin. The analysis will not only produce predictions of nominal design performance, but will also address uncertainties in performance due to variability in design parameters, material properties, and the environment.

The focus of this paper is on structural-thermal-optical integrated performance analysis for JWST. The STOP analysis process will be described, followed by representative results based on models representing the design at the time of the system requirements review (SRR). The paper concludes with a discussion of planned STOP analysis sensitivity studies.

\section{STRUCTURAL-THERMAL-OPTICAL (STOP) ANALYSIS PROCESS}

\subsection{Overview}

The performance budget dictates that the opto-mechanical stability of the JWST observatory be maintained within specification between recalibrations. The current performance budget allocates $25 \mathrm{~nm}$,rms WFE for alignment drift and $32 \mathrm{~nm}$,rms for figure drift. This leads to a RSS total allocation of $41 \mathrm{~nm}, \mathrm{rms}$ for drift stability. One of the uses of STOP analysis on the JWST project, and the focus of this paper, is to estimate alignment figure drift due to thermal distortions resulting from re-pointing (slewing) of the observatory. The multi-disciplinary STOP analysis process described here links thermal, structural, and optical models in a "bucked-brigade" fashion to predict the response of the system to a slew maneuver about the pitch axis. The process is outlined schematically in Figure 2 . The focus of current analyses is to predict the change in optical performance due to a "worst-case" slew between the hot and cold observatory orientations. This slew case results in the maximum thermal distortion and hence bounds the expected performance range. The process begins with a thermal analysis to determine steady-state temperature distributions for a range of 
observatory orientations. The orientations corresponding to hot and cold cases are identified, and temperature results are "mapped" from the thermal to the structural model. The structural model is then used to predict distortions resulting from thermal loading, and displacements of optically important surfaces are "mapped" to the optical model. Finally, perturbations from the structural analysis results are introduced to the optical model and the optical performance of the system is predicted. Further details regarding the discipline models and analyses are provided in the following sections. The models described here were developed by the prime contractor team and delivered to the government team at the time of the observatory system requirements review.

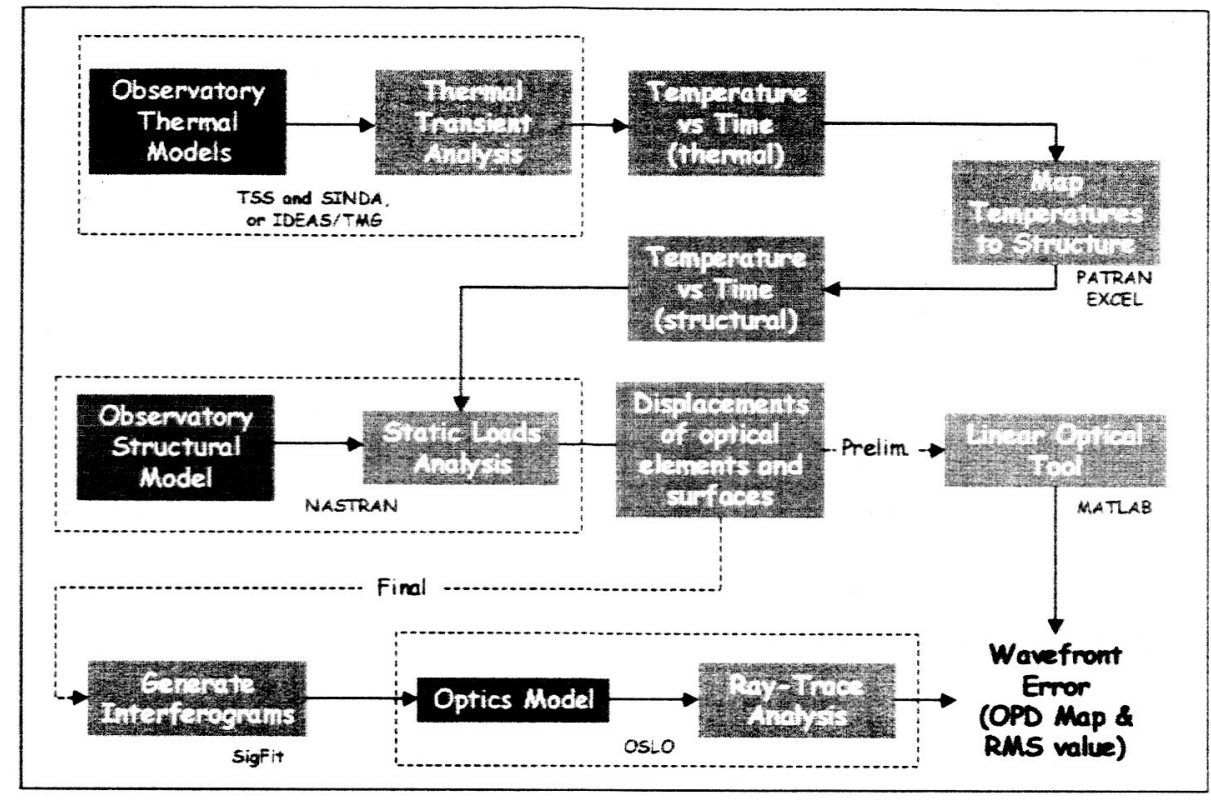

Figure 2: STOP analysis process map

\subsection{Thermal model}

The JWST observatory thermal model consists of a TSS radiation model and a SINDA/G thermal network math model. The TSS model consists of 3 major parts. The first part is the geometry file. This file defines shape, size, location, and surface properties. The second part is the radk file. By using the geometry file and ray tracing methods, radiative conductors are calculated and generated. The last part of TSS is a heat rate run. This file calculates and generates heat rates related to solar and infrared energy. Thirteen different cases were considered based on the sun angles corresponding to different observatory orientations. Eleven cases are based on sun angle $\left(+5^{\circ}\right.$ to $\left.-45^{\circ}\right)$ based on rotating about pitch axis. The two cases are based on sun angle $\left(+/-5^{\circ}\right)$ around the roll axis. SINDA/G is used to predict the temperature of the system using data set from the TSS runs, user generated linear conductive coupling, and necessary heater/power dissipations. Only the steady state response is considered here.

The geometry of the thermal model consists of several components. The first component is cold external geometry of the JWST observatory as shown in Figure 3. The second part is internal geometry that includes details for the primary mirror (PM) and the backplane (BP) assembly. The third major component is the 5 layers of sunshield. The fourth component is hot side of JWST that includes sunshield layer 1, solar array, bus and closeouts. The final piece of the component is internal details of deployable tower. Note that the optical properties used in all thermal models are end of life (EOL) properties.

Based on temperatures obtained from the thermal math model and the model topology contained in the geometric thermal model, a table of coordinates and corresponding temperatures is obtained for hand-off to the structures discipline. There are several approaches available for mapping the thermal model results to the structural model. One approach is to fit a function to the thermal results, then map this function to the structural model mesh. Another 
approach pairs corresponding thermal and structural model nodes (within a specified geometric tolerance) and assigns these nodes the same temperatures. These temperatures are then prescribed as "seeds" in a steady-state heat transfer analysis performed using the finite element model that provides a complete set of nodal temperatures for the structural analysis. Thermal mapping was accomplished via the first process for the analysis discussed in this paper.

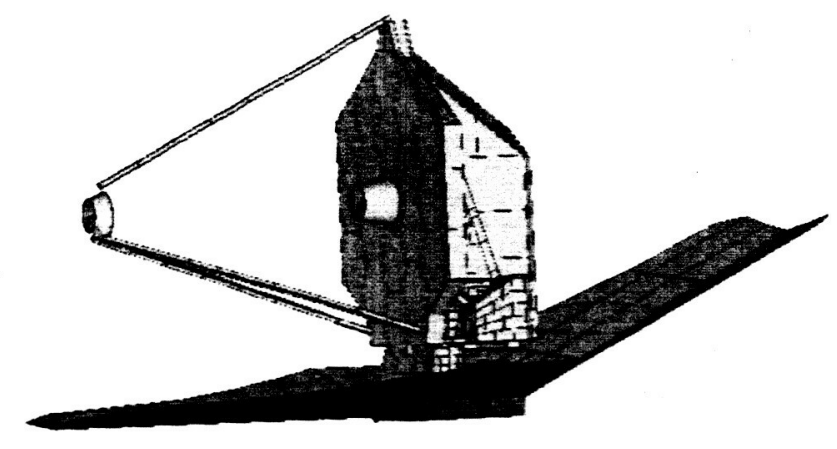

Figure 3: Thermal model

\subsection{Structural model}

The NASTRAN structural model of the deployed observatory is shown in Figure 4 . The fidelity of the model, which has approximately $\mathbf{4 0 0 0 0}$ degrees of freedom (DOFs), is appropriate for this stage of the project. It contains sufficient detail in the optical support structure areas to represent important thermo-structural effects without undue detail that would slow trade studies. One-dimensional beam elements are used to model the backplane, backplane support frame, and ISIM bench. Note that in actuality the OTE and ISIM metering structures are entirely formed of composite orthotropic material systems. Subsequent models will account for the thermal-structural response characteristics in all principle material directions. The primary mirror segment models are sufficiently detailed to capture critical resonant modes but are not appropriate for detailed stress analysis. Currently a single temperature is calculated for each segment in the thermal models, therefore more detailed structural models would not be useful. The mathematical validity of the STOP model is assured by means of a series of standard model check analyses. Unit gravity loading in each axis, unit-enforced displacement (and rotation) in each axis, ungrounded normal modes, and unit temperature change checks were run on the model.

Temperature-dependent values for the coefficient of thermal expansion are included in the model. NASTRAN requires that the tabulated values are "secant" CTE values. Element thermal strain is calculated by multiplying a single CTE table entry by the difference between the applied temperature and the material reference temperature. The thermal strain behavior of all materials is modeled in this way. This implementation of temperature-dependent thermal strain in NASTRAN complicates analyses between two on-orbit states, such as a slew to a new observation target. Two successive linear static analysis runs, both from the reference temperature state, are required. The applied temperature loads are the initial and final on-orbit temperature states. The difference between these two states represents the effect of the slew. The differences between states are calculated outside of NASTRAN, such as in pre- and post-processing software or MATLAB.

Structural deformations are input as perturbations to the optical model to assess optical performance. Mappings were required between the structural model and two different optical models (see discussion in the following section): optical ray trace model and linear optical model. An interferogram file is used as the interface between the NASTRAN structural model and the OSLO optical ray trace model for mapping primary mirror deformations. The interferogram file is generated using SigFit software developed by Sigmadyne, Inc. (Version 2003-R2). The interferogram (or hitmap) is a rectangular array of data over an optical surface onto which surface deformations are interpolated. Note that for this analysis a single interferogram was generated for the entire primary mirror and applied as a perturbation to an optical model with a monolithic primary mirror. Alternately, interferograms could be generated for each segment of the 
primary mirror assembly and used as perturbations in a segmented optical model. Additionally, since the current structural model only provides predictions for optical surface deformation on the primary mirror, rigid body motions are provided to the optical analyst for the secondary mirror, tertiary mirror, fine steering mirror, and image plane. The interface between the structural model and the linear optical model is a matrix of six degree of freedom motions, three translations and three rotations, for 22 optics (18 primary mirror segments, secondary mirror, tertiary mirror, fine steering mirror, and image surface).

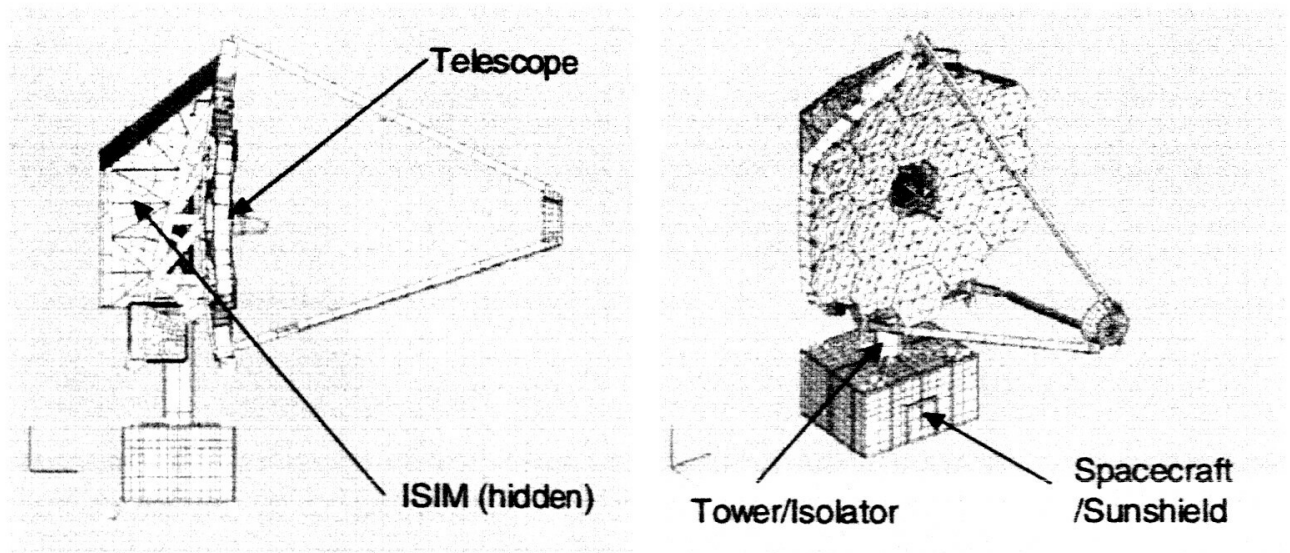

Figure 4: Structural model

\subsection{Optical models}

The JWST government analysis team uses two different models to assess optical performance. The first is an optical ray trace model, and the second is a linear optical model that is used primarily for first-order perturbation studies. The following sections describe each of the models in detail.

\subsubsection{Optical ray trace model}

Ray tracing for this analysis is performed using either of two optical prescriptions delivered from the prime contractor: one having a monolithic primary mirror surface, and the other having a segmented primary (non-sequential surface). The former is generally used for analyses that assume the primary mirror is aligned perfectly, while the latter is used to more accurately model alignment procedures and other more detailed analyses. Both models are delivered in OSLO optical design software lens format. These delivered models are modified slightly to decouple the coordinate systems between optical surfaces to convert the sequential layout coordinates to a global coordinate system. This allows each surface to be moved independently with respect to all other surfaces, so that any secondary mirror motion, for example, does not also move the tertiary mirror and other follow-on surfaces.

Rigid body perturbations due to thermal (or other) loads are applied to either optical model by changing the coordinates defining the location of each optic, while deformations to the optical surfaces are applied to each surface using an interferogram file, a grid of normal displacements from the optical surface. When the monolithic primary mirror model is used, a combined interferogram of all primary mirror segments are contained within a single file, which then simulates a segmented model to the level of the grid spacing of the interferogram.

The primary figure of merit for this analysis is root-mean-square wave-front error (RMS WFE), evaluated at the exit pupil of the system for the central field point. This is also referred to as a map of optical path differences, or "OPD map". If the rate of change of the perturbation is much slower than the exposure time or the ability for the fine guidance system to track the image, as it will be for typical thermal influences, then the RMS WFE is evaluated with the best-fit plane removed. This figure of merit is calculated in OSLO, but we also export the opd map into MATLAB for further analysis and archiving. 


\subsubsection{Linear optical model}

The approach taken for the linear optical model involves performing all ray tracing in advance through the system with a single known rigid body perturbation applied to each surface and each degree of freedom. This generates linear sensitivities, which are used as transfer matrices converting rigid body motions of optical components to absolute image motion at the detector and wave front error induced due to the misaligned components. The linear optical model can be briefly described as a first-order Taylor expansion of the optical path lengths of a grid of rays traced through the system, uniformly spaced at the entrance pupil. The variables expanded upon are the rigid body degrees of freedom (DOF) for each optical component in the system. Naturally, the model is valid in the linear approximation only for small perturbations of the rigid body degrees of freedom. This can be summarized in the following equation:

$$
L=L_{0}+\frac{\partial L}{\partial U} \Delta U+O(2)
$$

where $\boldsymbol{L}$ contains the optical path lengths of the grid of rays from the entrance pupil to the exit pupil, $L_{0}$ contains the nominal (unperturbed) path lengths for the system, $\Delta \boldsymbol{U}$ represents the perturbation of the rigid body degrees of freedom, and $\boldsymbol{O}(2)$ represents the second and higher order terms of the expansion which are disregarded for this linear analysis. The first-order derivative term, $\frac{\partial L}{\partial U}$, is constructed by perturbing each DOF, one at a time, and evaluating the change in path lengths from the nominal for each case. This process is described in detail in Ref. 1. Note that this firstorder derivative term contains all wavefront data including absolute piston, tip/tilt, and all higher-order terms, since it is evaluated with respect to the nominal reference sphere at the exit pupil without regard to chief ray motion. Wavefront error is defined here as the optical path length difference, or OPD, between the perturbed system and the nominal system where all path lengths are equal to that of the chief ray. Comparing wave front maps generated from Eq. (1) to those generated by ray tracing show less than $1 \%$ difference for random realizations of perturbations of all components up to 1 micron or micro-radian motions or tilts, respectively.

Structural analysts evaluate $L$ by determining elements of $U$ resulting from deformations calculated in NASTRAN, scaling the $\frac{\partial L}{\partial U}$ matrices by these vector components; and summing the products. The root-mean-square (RMS) value of $\mathrm{L}$ is the key performance metric, although others are used. A number of MATLAB functions and scripts have been created to facilitate the wavefront error calculation process. Among the capabilities added by these scripts are: minimization of RMS OPD with the fast-steering mirror; slew maneuver modeling from the results of two NASTRAN runs, including the ability to remove reference states; automated NASTRAN results plotting with performance metric labeling; and decomposition of OPD results into spatial frequency components.

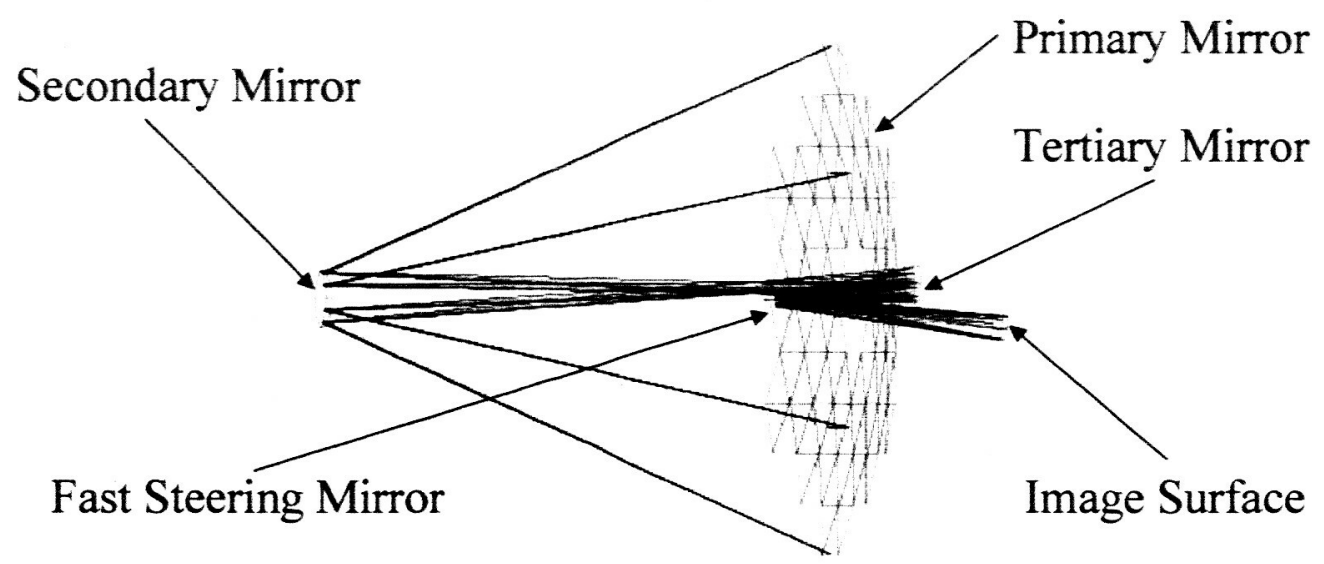

Figure 5: Optical model 


\section{STOP ANALYSIS RESULTS FOR JWST AT SRR}

\subsection{Thermal model results}

Figure 6 presents the steady-state temperature profile for the hot case $\left(-15^{\circ}\right.$ pitch angle) illustrating primary mirror (PM) and backplane (BP) structure temperatures. The hot case is defined by the hottest average PM temperature among the cases studied. The plot indicates that for the hot case the maximum PM temperature is $58.3 \mathrm{~K}$ and the minimum temperature is $35.2 \mathrm{~K}$. Note that the PM temperature gradient is primarily in the V3 direction with the hot side nearest the sunshield. Also note that the backplane structure has a temperature gradient that is similar albeit at a slightly higher temperature than the PM. Of interest in assessing the operational WFE stability of the telescope is the worst case temperature change as the observatory slews about the pitch axis. Figure 7 presents a plot of the worst case delta temperature profile (hot case - cold case), where the cold case is for a $-45^{\circ}$ pitch angle. The average change in temperature between states is approximately $0.3 \mathrm{~K}$. The maximum change in temperature between states is less than 0.5 $K$ over the entire PM and BP with the peak change occurring nearest the sunshield.

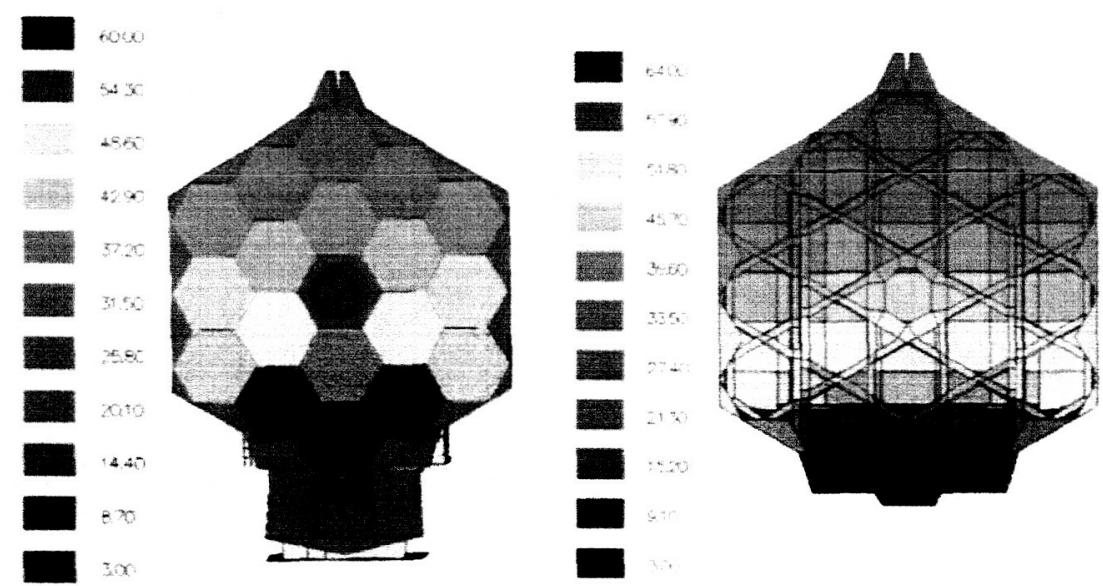

Figure 6: Temperature profile ('Hot' case) plot

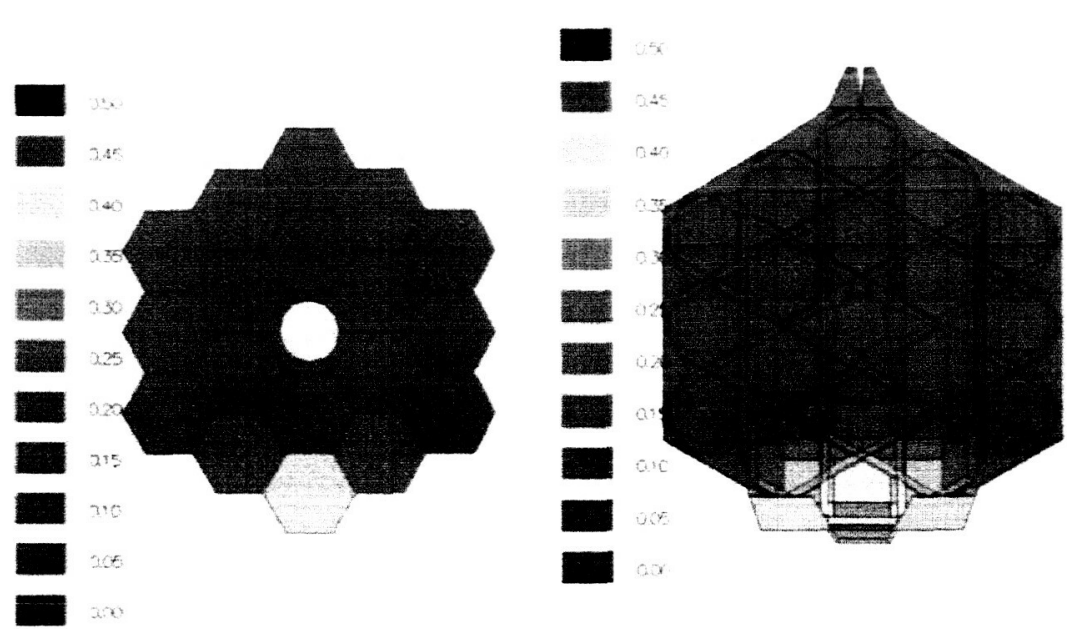

Figure 7: Delta temperature profile ('Hot-Cold' case) plot 


\subsection{Structural model results}

Figure 8 presents results from the structural model illustrating the deformations resulting from a change in temperature corresponding to the worst-case slew defined by the thermal analysis. Peak total displacements are on the order of 200 $\mathrm{nm}$ and occur in the secondary mirror support structure. The magnitude of the total displacements exhibited by the primary mirror (primary mirror segments plus backplane support structure) is on the order of $50 \mathrm{~nm}$. Of interest in assessing wavefront error are the primary mirror surface normal displacements. NASTRAN results were post-processed using the Sigfit software package to obtain surface normal displacements with rigid body motions removed. The RMS value of the surface normal displacements is $8 \mathrm{~nm}$ and the peak-to-valley value is $42 \mathrm{~nm}$.

The structural model predicts that while the backplane support structure is the main contributor to primary mirror deformations, the PMSA's also make a significant contribution. Analysis shows that the deformations of the individual PMSA's are primarily due to thermal strains in the primary mirror support and actuation system with lesser contributions from the mirror substrate. The deformations result in a rigid-body-like pistoning of each of the segment mirror substrates with negligible surface deformation. Note that since the change in primary mirror temperature between states is greater near the sunshield, the PMSA's on that side of the primary mirror undergo larger motions than the PMSA's opposite the sunshield.
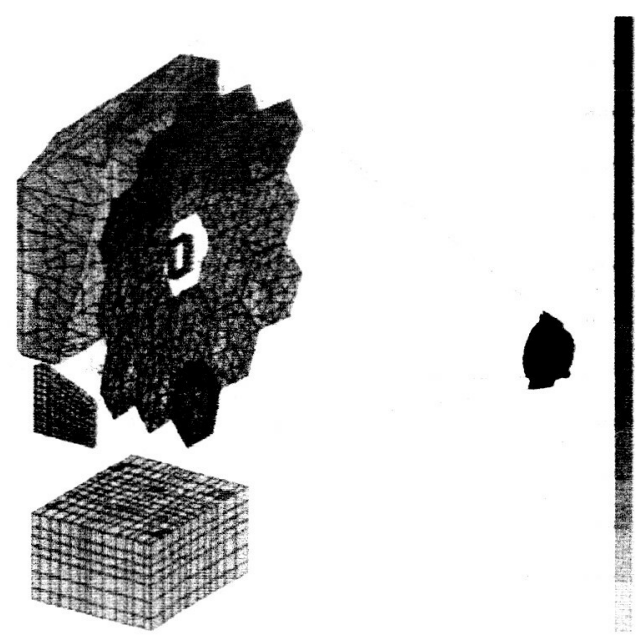

Figure 8: Structural deformation plot

\subsection{Optical model results}

Results from the optical models with perturbations based on structural deformations resulting from the worst-case slew case are presented in this section. Optical performance predictions for RMS WFE are broken down into three spatial frequency bands: low ( $<5$ cycles per aperture), mid $(>5$ and $<35$ five cycles per aperture), and high $(>35$ cycles per aperture). These contributions are calculated in such a way that when RSS's they equal the total WFE. Optical results below are presented as total RMS WFE followed by the frequency decompositions.

Figures 9 and 10 present maps of the optical path difference evaluated at the exit pupil of the system for the central field point. The OPD map from the optical ray trace model, Fig. 9, shows an RMS WFE of $18.2 \mathrm{~nm}$ (Low=17.8 nm, Mid=6 $\mathrm{nm}$, High=2 $\mathrm{nm}$ ) and a peak-to-valley WFE of $89 \mathrm{~nm}$. The OPD map from the linear optical model, Fig. 10, shows an RMS WFE of $14 \mathrm{~nm}$ (Low=13.2 nm, Mid=4.6 nm, High=1.6 nm) and a peak-to-valley WFE of $72 \mathrm{~nm}$. Overall, results from the two optical models show good agreement. Qualitatively the OPD maps are very similar, and the RMS WFE predicted by the linear optical model is within $20 \%$ of that predicted by the ray trace. As noted in the structural results section, the two main contributions to WFE are deformations in backplane support structure and the PMSA's. On a global scale, the OPD is dominated by a power aberration associated with deformations in backplane support structure. 
Examination of the WFE by spatial frequency band shows that, as expected, the WFE is dominated by a low spatial frequency contribution.

The analytical results from the ray trace and linear optical models show that the observatory currently meets an RSS total allocation of $41 \mathrm{~nm}, \mathrm{rms}$ for drift stability with ample margin. Future studies, outlined in the following section, will explore the sensitivity of these nominal results.

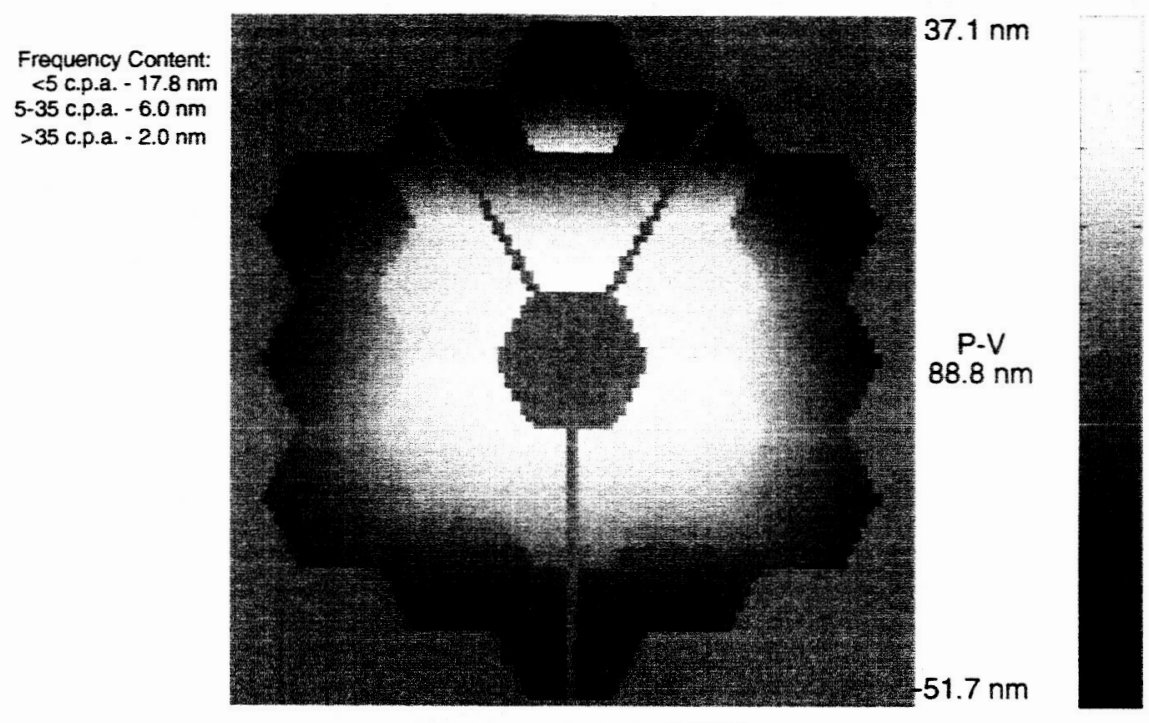

$18.2 \mathrm{~nm}$, RMS WFE

Figure 9: OPD map plot from OSLO ray trace

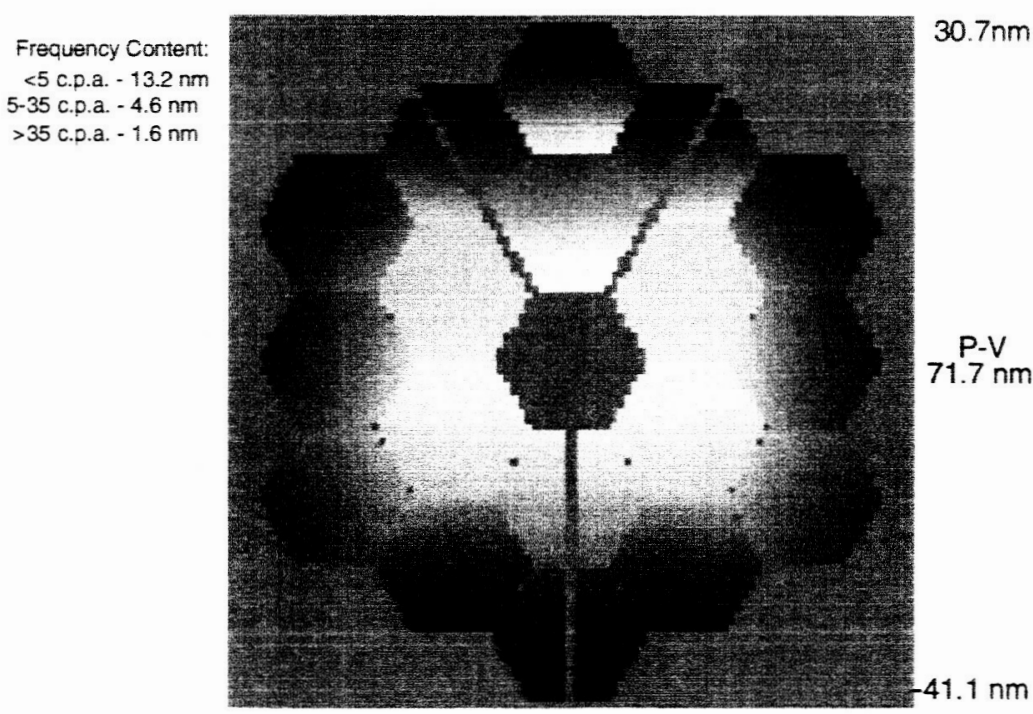

$14.0 \mathrm{~nm}, \mathrm{RMS}$ WFE

Figure 10: OPD map plot from linear optical model 


\section{SENSITIVITY STUDIES}

The sensitivity of observatory performance to variability in key parameters such as material properties and the environment will be studied using Monte Carlo statistical models using changes in wavefront error as the scalar parameter to correlate. The models will be applied at various levels of refinement to gain insight to the overall system behavior and to define key parameters to consider in the analysis. The analysis flow will follow a process along the lines of the following:

1. Define benchmark temperature cases to represent maximum expected disturbance inputs. Currently the benchmark disturbance case is the worst-case slew between hot and cold pointing conditions.

2. Identify key variable parameters and a statistical model for each parameter. Expected parameters to consider include coefficient of thermal expansion, thickness variations, and stiffness variations.

3. Determine the sensitivity matrix of WFE to each of the parameters by developing math models (engineering formulas, finite element models, optical models, etc.) and studying the influence of each parameter on the monitored response variable. Initial development of the sensitivity matrix will assume the parameters are uncorrelated and can be linearly combined.

4. Generate random sets of each key parameter.

5. Combine each random set with the sensitivity matrix and calculate a random sampling of WFE due to variations in key parameters.

6. Calculate the statistics (mean and variance) of the WFE variation and compare to the system requirements.

Variations of this approach will be used to study several items of interest including but not limited to the following:

- Determine at the level of spatial variation for each parameter that needs to be considered. For example, does the representation of the composite tubular elements in the OTE and ISIM structures require consideration of all three material orthotropic axes? Is it sufficient to represent each tubular element with a uniform local average or does the spatial variation need to be modeled at each element? These studies will support defining the level of model fidelity required.

- Study the effects of shifts or bias in the expected versus delivered nominal set point for each parameter assuming the same variance model.

- Study the impacts of localized bias in the assembled telescope elements. For example, the backplane center section and wings could be fabricated from three different lots of composite prepreg. Also, the length to which each actuator is extended will affect the athermalization for each bipod actuator set on each PMSA. These conditions open the door for localized variations or bias in the nominal response of each parameter model.

- Support manufacturing and process definition by evaluating various statistical models for each parameter, defining the allowable variation, and identifying key parameters on which to monitor and control the process.

- Study the effect of uncertainties in the thermal models and support the establishment of the required levels of accuracy in thermal performance predictions.

- Assess the level of uncertainty in nominal STOP analysis performance predictions.

Statistical models for thermal distortion of the observatory will be a valuable tool employed over the life of the project. Initial models of low fidelity have already supported system requirements definition. Detailed component level models are anticipated in support of each major review cycle, and an appropriately detailed system level model is required to accomplish analysis on which the verification plan is anchored. The validity of these models will be supported by testing and correlation activities at the material, component, and system levels.

\section{SUMMARY}

An overview of the integrated modeling process used by the JWST government team to predict the on-orbit thermoelastic stability of the observatory has been described. The multi-disciplinary analysis process involves linking thermal, structural, and optical models to predict end-to-end performance. Results were presented for models representative of the observatory concept at the time of the system requirements review in late 2003. Predictions from this analysis for nominal performance indicate that the observatory concept meets current requirements for on-orbit stability. An approach for assessing the sensitivity of the nominal performance due to variations in key parameters such as material properties and thermal loading was outlined. Future studies will implement this process to support the further evolution of the observatory design. 


\section{REFERENCES}

1. Joseph M. Howard, "Optical modeling activities for the James Webb Space Telescope (JWST) project: I. The linear optical model," Proc. SPIE Int. Soc. Opt. Eng. 5178, 82 (2004).

2. Howard, J.M., et al, "Optical Modeling Activities for the James Webb Space Telescope (JWST): 2. Determining Image Motion and Wavefront Error Sensitivities for a Segmented Optical System Over An Extended Field," SPIE Astronomical Telescopes and Instrumentation Conference, June 2004, SPIE 5487-142.

3. Hyde, T.H., et al, "Integrated Modeling Activities for the James Webb Space Telescope (JWST): Optical Jitter Dynamics Analysis," SPIE Astronomical Telescopes and Instrumentation Conference, June 2004, SPIE 5487-122.

4. Nella, J., "James Webb Space Telescope (JWST) observatory architecture and performance," SPIE Astronomical Telescopes and Instrumentation Conference, June 2004, SPIE 5487-23.

5. Mosier, G., Parrish, K., Femiano, M., Redding, D., Kissil, A., Papalexandris, M., Craig, L., Page, T., and Shunk, R., "Performance Analysis Using Integrated Modeling", NGST Monograph No. 6, August 20006.

6. Mosier, G., Femiano, M., Ha, K., Bely, P., Burg, R., Redding, D., Kissil, A., Rakoczy, J. and Craig, L., "An Integrated Modeling Environment for Systems-level Performance Analysis of the Next Generation Space Telescope," SPIE Vol. 3356, Space Telescopes and Instruments V, 1998. 